\title{
54684: Mechanisms Involved in Trichloroethylene-Induced Liver Cancer: Importance to Environmental Cleanup
}

(First Year of Funding: 1996)

DOE report Number:

$3 / 1 / 00$

Principal Investigator

Richard J. Bull, Ph.D.

Pacific Northwest National Laboratory

P.O. Box 999, MSIN P7-56

Richland, WA 99352

(509) 373-6218 (phone)

Dick.bull@pnl.gov

Co-Principal Investigator

Brian D. Thrall

Pacific Northwest National Laboratory

P.O. Box 999, MSIN P7-56

Richland, WA 99352

(509) 376-3809 (phone)

Brian.thrall@pnl.gov

\section{Contributors}

Lyle B. Sasser (Lyle.sasser@pnl.gov)

John H. Miller (John.h.miller@pnl.gov)

Irvin R. Schultz (Irvin.schultz@pnl.gov)

\section{Graduate Students}

Anja J. Stauber

Alberto Gonzalez-Leon

Junko Kato-Weinstein

James Merdink

Melissa K. Linghor 


\section{Problem Statement:}

The objective of this project is to develop critical data for improving risk-based clean-up standards for trichloroethylene (TCE).

Importance to DOE: Clean-up costs for chlorinated solvents found on DOE sites are most frequently driven by TCE because it is both the most wide spread contaminant and is generally present at the highest concentrations. Data that would permit non-linear approaches to the development of standards for TCE would reduce complex-wide clean up costs by hundreds of millions of dollars. Moreover, precedence will be established that will allow more accurate estimates of risk to be developed for other environmental contaminants.

Current regulatory actions that research will impact: EPA is currently reviewing its risk assessment for TCE. One of us (RJB) has worked with EPA on this review by writing the mode of action section for liver tumor induction for their determination.

\section{Research Objectives}

The project is organized around two interrelated tasks:

Task 1 develops the basic dosimetry parameters and provides in vivo data describing the mode of action tumorigenic and for the metabolites of TCE that produce liver cancer in mice, dichloroacetate (DCA) and trichloroacetate (TCA). Early work suggested that TCA was primarily responsible for TCE-induced liver tumor. More recent, mechanistic observations indicated that DCA played a prominent role. Therefore, studies were designed to determine whether the effects of DCA were mediated through a mode of action that affects primarily tumor growth rates.

Task 2 seeks specific evidence that TCA and DCA are capable of promoting the growth of spontaneously initiated cells from mouse liver, in vitro. The data provide the clearest evidence that both metabolites act by a mechanism of selection rather than mutation. These data are necessary to select between a linear (i.e. no threshold) and non-linear low-dose extrapolation models.

\section{Research Progress and Implications}

Tumorigenesis studies: Originally, TCA was considered the active metabolite in inducing liver cancer with TCE. We have now sequenced codon 61 of the $\mathrm{H}$-ras protooncogene in a total of 30 TCA-induced and 64 DCA-induced liver tumors. In the past year we sacrificed 47 mice that had been treated with $1 \mathrm{~g} / \mathrm{kg}$ TCE in an aqueous emulsion (Alkamuls ${ }^{\circledR}$ ) by gavage for 79 weeks. Forty-four tumors were recovered from these mice.

Sequencing of codon 61 of the $\mathrm{H}$-ras gene has now been completed for all three chemicals. Lower mutation frequencies seen with DCA and TCE in aqueous vehicle and this is much more consistent with observations made with other tumor promoters. The mutation frequency observed with TCA is much higher than observed with other peroxisome proliferators. The mutation spectra within tumors carrying a H-ras mutation are compared across compound, the results with DCA are more similar to that of TCE than that of TCA, suggesting that DCA does contribute to the tumor response.

Since TCA has been shown to be a peroxisome proliferator and that is a mechanism that questionable relevance for humans, our work has focused on DCA. We have used magnetic resonance imaging of tumors to demonstrate that cessation of exposure to DCA causes tumors to stop growing. Modeling of these data indicates that these data indicates that effects of DCA on cell replication rates and apoptosis can completely account for the apparent "initiating" activity of DCA. Therefore, DCA and by extension, TCE, belong to a category of liver carcinogen that should be treated with non-linear methods to estimate safe doses.

Pharmacokinetic results: Issues related to the dosimetry of the DCA that is produced from the metabolism of TCE have become clearer in the past year. There are two issues, 1) the 
amount of DCA that is generated from a given dose of TCE and 2) the amount of DCA that must be in the liver to induce a carcinogenic response. Data generated in the past year indicates that the area under the curve (AUC) projected for DCA from TCE based on its pharmacokinetic properties indicates a level that would be expected to give rise to a tumor response.

Establishing selective advantage as a mode of action for DCA and TCA: The assay was used to measure the relative potency of DCA and TCA to promote the clonal growth of initiated hepatocytes. TCA and DCA were shown to increase the growth of colonies on soft agar at concentrations of these metabolites that approximate those seen in vivo. The colonies produced with TCA and DCA reflected the different phenotypes of the tumors they induce in vivo.

In the past year, we have been able to simulate the effects of DCA in vivo on glycogen accumulation in isolated primary hepatocytes. We yet to establish the mechanism for this accumulation and its relationship to the tumorigenic response. However, the demonstration of the growth response and glycogen accumulation suggests that mechanistic studies of DCA's effects can be conducted in vitro with some confidence that effects are reflecting in vivo effects of the chemical.

\section{Planned Activities}

The PI has been working with the National Center for Environmental Assessment of EPA in developing the Symposium at the 2000 Annual Society of Toxicology Meeting that will lay out EPA's findings. We were asked to summarize the state of the mode of action information data that is available on TCE and its metabolites on liver tumor induction. These data will form the basis for modification of the current MCL for TCE in drinking water. It is anticipated that this will require pressure on the OGWDW to put TCE on their regulatory calendar.

A renewal of this proposal is being prepared.

\section{Manuscripts (FY00):}

Barton, H.A., Bull, R., Schultz, I., Andersen, M.E. (1999) Dichloroacetate (DCA) dosimetry: interpreting DCA-induced liver cancer dose response and the potential for DCA to contribute to trichloroethylene-induced liver cancer. Toxicology Lett. 106:9-21.

Gonzalez-Leon, A., Merdink, J.L., Schultz, I.R., Bull, R.J. (1999) Effect of pretreatment with dichloroacetate or trichloroacetate in drinking water on the pharmacokinetics of a subsequent challenge dose in B6C3F1 mice. Chem.-Biol. Interactions 123:239-253.

Miller, J.H., Minard, K.R., Wind, R.A., Orner, G.A., Sasser, L.B. and Bull, R.J. (2000) In vivo MRI measurements of tumor growth induced by dichloroacetate: Im plications for mode of action. Toxicology, In press.

Bull, R.J. (2000) Mode of action of liver tumor induction by trichloroethylene and its metabolites, trichloroacetate and dichloroacetate. Environ. Health Perspect. In press.

Kato-Weinstein, J., Stauber, A.J., Orner, G.A., Thrall, B.D. and Bull, R.J. (2000) Differential effects of dihalogenated and trihalogenated acetates in the liver of B6C3F1 mice. J. Appl. Toxicol. Accepted with revisions

Merdink, J.L., Bull, R.J. and Schultz, I.R. (2000) Trapping and identification of the dichloroacetate radical from the reductive dehalogenation of trichloroacetate by mouse and rat liver microsomes. Submitted

Merdink, J.L., Bull, R.J. and Schultz, I.R. (2000) Toxicokinetics of bromodichloroacetate in B6C3F1 mice. Submitted 